\title{
Pathogenesis of atherosclerosis in peripheral artery disease for diabetes patients
}

\author{
Pavelina Mihaela ANDREI ${ }^{1}$, Maria Daniela TANASESCU ${ }^{1,2}$, Alexandru MINCA $^{1,2}$, \\ Andra-Elena BALCANGIU-STROESCU ${ }^{3,4}$, Daniela BALAN ${ }^{3}$, Andrada $\mathrm{MIHAI}^{1,5}$, \\ Alexandra Maria LIMBAU $^{1}$, Constantin IONESCU TIRGOVISTE ${ }^{1,5}$ \\ ${ }^{1}$ Faculty of Medicine, "Carol Davila" University of Medicine and Pharmacy, Bucharest, Romania \\ ${ }^{2}$ Department of Internal Medicine, University Emergency Hospital, Bucharest, Romania \\ ${ }^{3}$ Faculty of Dental Medicine, "Carol Davila" University of Medicine and Pharmacy, Bucharest, Romania \\ ${ }^{4}$ Department of Dialysis, Emergency University Hospital, Bucharest, Romania \\ 5"N.C. Paulescu“ National Institute of Diabetes, Nutrition and Metabolic Diseases, Bucharest, Romania
}

\section{ABSTRACT}

Over 170 million people worldwide are diagnosed with diabetes and it's estimated that the number will reach 370 million cases by 2030. Diabetes represents a major risk factor for all cardiovascular diseases with a high mortality rate. Furthermore, diabetes represents a risk factor for peripheral artery disease (PAD) cases and its association leads to significant increase in risk for vascular and cardiac events at foot and leg level thus impacting the quality of life of the patient. The atherosclerosis which is found at the base of PAD pathogenesis results from accumulation of lipids and fibrous material between the tunica intima and tunica media vascular walls resulting in progressive constriction of the vessel's flux. This phenomenon can lead to complications such as thrombosis or rupture of the atheroma plaque which results in occlusion of downstream vessels. Reducing the risk factors which contribute to the atherosclerosis pathogenesis such as endothelial dysfunction, increase in platelets activity, dyslipidemia, immunological and inflammatory factors as well as tobacco contributes to the reduction of atherothrombotic episodes with impact on the patient's quality of life
\end{abstract}

Keywords: atherosclerosis, peripheral artery disease, diabetes mellitus

\section{INTRODUCTION}

Diabetes mellitus is a significant risk factor for PAD, followed by smoking, old age, dyslipidemia and hypertension. Diabetes mellitus is characterized by a chronic hyperglycemic status, insulin resistance and dyslipidemia (1). Metabolic changes which are at the base of diabetes contribute to vascular inflammation, endothelial dysfunction, platelets activation, vessel constriction and heightened thrombotic risk, conditions which are involved in the PAD pathogenesis in diabetic patients (2).

PAD prevalence is significantly higher among diabetes patients compared to the general population. In 
the diabetic population the association of the diabetic neuropathy underestimates the PAD prevalence due to the less severe symptoms. The PAD pathogenesis at diabetic patients is identical to non-diabetic patients, the difference being that PAD in the case of diabetics is potentiated by the presence of diabetes itself (3).

PAD patients show change in lipidic metabolism which involves increased levels of triglycerides, cholesterol, lipoprotein (a), apolipoprotein B and VLDL in conjunction with decrease in lipoproteins with defensive roles such as HDL, apolipoprotein A-I and apolipoprotein A-II, phenomenon which contributes to the increased risk of cardiovascular disease. Lipoprotein (a) is an independent risk factor for PAD. This is genetically determined. In the Quebec Cardiovascular Study the risk of intermittent claudication was double for men which presented high concentration of lipoprotein (a) compared to women (4).

Optimization of the glycemic control for diabetes patients also maintains a normal plasmatic concentration of cholesterol and triglycerides (5). The dyslipidemia in diabetes mellitus is represented in particular by hypertriglyceridemia and not by hypercholesterolemia which is the frequent cause for nondiabetic patients (6). A particularity of the dyslipidemia associated with diabetes is the change in qualitative proprieties of lipoprotein in the case of a normal lipid profile, which could explain the heightened vascular affection for diabetes patients. The lipoprotein (a) also participates in the atherogenic process.

\section{DYSLIPIDEMIA}

In diabetes mellitus the insulin deficit or insulin resistance determine the reduction of the lipoprotein lipase (LPL) activity which leads to the decrease of the chylomicrons catabolism and VLDL particles as well as to the conversion rate of VLDL - LDL. The consequence of heightened activity of the hormone-sensitive lipase adipocyte (HSL) contributes to an increase of the free fatty acids levels at hepatic level and also contributes to the increase of apoprotein B100 and VLDL synthesis. The activity of the cholesterol esters transfer protein (CETP) is also increased. Insulin resistance determines the reduction of LDL receptors which leads to reduction in LDL catabolism hence further contributing to the appearance of hypertriglyceridemia (7). Small and dense particles of LDL in high concentration reduce the LDL receptors affinity thus increasing the oxidation susceptibility. LDL has a major contribution in the acceleration of atherogenesis for diabetic patients. A $10 \%$ increase in LDL levels is associated with a $20 \%$ increase in risk of cardiovascular events.

In diabetic population the decrease of HDL is seen with a frequency of $30 \%$ in comparison $15 \%$ for nondiabetics. Increased HDL catabolism is caused by the in- crease in the activity of cholesterol ester transfer protein (CEPT) due to hyperglycemia, as well as due to the increased activity of the hepatic lipase (HL). A cholesterol value greater than $150 \mathrm{mg} / \mathrm{dl}(3.9 \mathrm{mmol} / \mathrm{l})$ shows a heightened incidence of atherosclerosis.

\section{LDL OXIDATION}

LDL increase in conjunction with HDL decrease represents important risk factors for atherosclerosis. The antiatherogenic effect of HDL is due to reverse transport of cholesterol from tissues to liver, as well as due to $L D L$ protection from oxidation through the antioxidative enzyme paraoxonase (PON1). HDL level has a reverse proportional relation with cardiovascular risk. Values over $75 \mathrm{mg} / \mathrm{dl}(1.9 \mathrm{mmol} / \mathrm{l})$ are associated to a low risk of cardiovascular complications and atherosclerosis.

Chronic hyperglycemia determines the glycosylation of apo B100 from LDL's structure resulting in glycosylated LDL which become sensitive to oxidation and which gain atherogenic properties. Plasmatic LDL contains antioxidants which are dissolved in lipidic core such as vi$\operatorname{tamin} \mathrm{E}$, beta carotene and ubiquinone which themselves can be oxidized hence they lose their protective role and they gain pro-oxidizing effects.

The oxidizing effects change of LDL is associated with a heightened affinity for macrophage scavenger receptors (CD 36 or scavenger receptor $B$ is the most important) from which it bounds at arterial wall level. LDL cholesterol intake by macrophages is initially an adaptive response which prevents the endothelial injury by the LDL. However, cholesterol accumulation in foam cells leads to mitochondrial dysfunction, apoptosis and necrosis resulting in activation of cellular proteases, proinflammatory cytokines and prothrombotic molecules (8). As a result, the chemotaxis for monocytes is increased through activation of $\beta 1$ monocytic integrin, the production of nitric oxide is reduced and the platelet aggregation is increased. Hence the increased level of oxidized LDL correlates with the atherosclerosis lesions severity which determines the role which it takes in the atheroma plaque instability (9).

Oxidized LDL cause changes at the surface level of endothelial cells, heightens susceptibility to apoptosis of smooth muscle cells, promotes inflammation through the cytokines released from macrophages and oxidized LDL antibody formation (10). Oxidized LDL antibodies titer can be used as both as a atherosclerosis predictor and as a plaque stability marker (11).

Certain receptors can have effect on macrophage differentiation such as peroxisome proliferator-activated receptor gamma (PPAR- $\gamma$ ) or glitazone receptor. This effect can have clinical implications, such as that 
thiazolidinediones (for example rosiglitazone), used on type II diabetes patients for increase the insulin sensibility, and activates PPAR- $\gamma$ hence affecting the atherosclerosis process.

Lipoprotein (a) promotes atherosclerosis and through bounding to the apo (a) molecule, a protein with a similar structure as the plasminogen, could interfere with fibrinolysis. It also inhibits the transformation of plasminogen into plasmin favoring thrombus creation. Lipoprotein (a) can lead to forming of foam cells through macrophage bounding (12).

For diabetic dyslipidemia the ADA recommended value of $L D L$ to be below $100 \mathrm{mg} / \mathrm{dl}(2.60 \mathrm{mmol} / \mathrm{l}), \mathrm{HDL}$ above $45 \mathrm{mg} / \mathrm{dl}(1.15 \mathrm{mmol} / \mathrm{l})$ for men and over $55 \mathrm{mg} /$ $\mathrm{dl}$ for woman, total cholesterol under $185 \mathrm{mg} / \mathrm{dl}(4.8$ $\mathrm{mmol} / \mathrm{l})$ and triglycerides under $150 \mathrm{mg} / \mathrm{dl}(2.7 \mathrm{mmol} / \mathrm{l})$.

\section{PATHOGENESIS OF ATHEROSCLEROSIS}

The atherosclerosis pathogenesis includes several factors such as: the aforementioned dyslipidemia, and diabetes, endothelial dysfunction, inflammatory or immunological factors, smoking as well as plaque rupture. Genetic predisposition also plays a role in plaque formation.

\section{Endothelial disfunction}

The endothelium represents an interface between blood and other tissue and has a role in the maintenance of vascular tonus and permeability as well as providing equilibrium between fibrinolysis and thrombosis. Imbalance of these functions can be involved in atherosclerosis. Besides diabetes, HTA and smoking the endothelial disfunction is caused by other factors such as increased homocysteine, reduction in nitric oxide synthesis and oxidized LDL participation (13). In atherosclerosis the endothelial dysfunction is an initial step in atherosclerosis and has a vasoconstrictor status, heightened vascular permeability for lipoproteins, and reduction of the triglyceride rich lipoproteins catabolism as well as a hyper coagulant status (14).

\section{The role of inflammation}

Histological observation shows inflammation since the early stages of atherosclerosis. The macrophages which have oxidized LDL intake release cytokines, intercellular adhesion molecule (ICAM-1), and monocyte chemotactic protein (MCP-1) and stimulate growth factors. MCP-1 promotes cellular adhesion to the endothelium (15). In normal physiological conditions MCP-1 is inhibited by the nitric oxide thus the atherogenic process is also inhibited. IL-1, IL-3, TNF- $\alpha$ are cytokines and stimuli for smooth muscular cells proliferation. TNF- $\alpha$ increases the permeability of en- dothelial cells, contributes to the forming of foam cells and induces macrophage differentiation. IL-1 and TNF- $\alpha$ present atherogenic effects.

IL-8 produced by neutrophils induces the proliferation and migration of endothelial cells and smooth muscle cells. The proinflammatory cytokine IL-1 $\beta$ has a major role in the appearance of atherosclerosis plaque. Proinflammatory cytokines induce cellular proliferation, production of oxygen reactive species, growth factor expression and stimulates matrix metalloproteinases (16).

IL-4 and IL-10 are antiatherogenic.

Increased $\mathrm{C}$ reactive protein represents an important marker in the atherosclerosis pathogenesis, since it can induce the expression of adhesion molecules in endothelial cells (16).

Lipoprotein associated phospholipase A2 (Lp-PLA2) is an enzyme secreted by macrophages which maintains the inflammation at skin level.

\section{Homocystein}

Homocystein in high concentrations which isn't caused due to genetic defects is associated with endothelial injury, reduced production of nitric oxide and hypercoagulation.

\section{Hypertension}

Hypertension represents a risk factor for atherosclerosis through its potential of increasing the arterial wall tension which can affect the repair process of arterial walls and contributes to aneurysm forming. Furthermore, the modified endothelium of hypertensive patients produce angiotensin II and endothelin with which have a vasoconstrictor role and the possibility of platelet aggregation and thrombosis (17). Angiotensin 2 stimulates lipooxygenase activity and LDL oxidation which contributes to local inflammatory response.

\section{Angiotensin 2}

Angiotensin 2 is correlated with atherosclerosis progression, especially when associated with hyperlipidemia. The macrophages from atherosclerotic lesions contain high quantities of angiotensin 2 which induces proliferation of smooth muscle cells as well as extracellular matrix production.

\section{Smoking}

Smoking changes the endothelium through the diminution of nitric oxide production, vasoconstrictor effect, increased level of fibrinogen and reduced fibrinolysis. Furthermore smoking causes increase in LDL oxidation and decrease the activity of the paraoxonase enzyme which protects against LDL oxidation (18). 


\section{Diabetes impact on atherogenesis}

Many studies show that a high concentration of insulin precedes the development of arterial lesions and contributes to the atherogenic effect alongside the dyslipidemia associated with diabetes. The insulin resistant state is either due to the increase of free fatty acids, IL-6 and TNF- $\alpha$ cytokines and the growth of hyperglycemic hormones such as cortisol, catecholamine, growth hormone and glucagon, either due to changes in the insulin receptor. Increase for circulating peptides such as amylin and leptin or reduction for peptides such as adiponectin and ghrelin are associated to the insulin resistance state. Insulin resistance is most often associated with obesity, dyslipidemia, arterial hypertension and cardiovascular complications (19).

Insulin resistance has proatherogenic effects through the proliferation potentiation of vascular smooth muscle cells and the synthesis of PAI-1 from these cells. These proatherogenic effects of insulin are mediated through the MAPK pathway (mitogen activated protein kinase) which shows that these changes of the vascular endothelium is caused by the blocking of signaling pathway of IRS 1 / IRS 2 (insulin receptor substrate) - PI3K (phosphoinositide 3-kinase). From a physiological perspective, insulin has vasodilation and antiatherogenic effects mediated through the PI3K signaling pathway (20).

Accelerated atherosclerosis in obese patients is caused by the increase of IL- $6, \mathrm{IL}-1 \beta$ and TNF- $\alpha$ cytokines, the increase of the procoagulant factors fibrinogen, PAI-1, factor VII, VIII - Von Willebrand factor, decrease of fibrinolytic factors, increase in small and dense $L D L$, increase of VLDL and apolipoprotein $B$ and decrease of HDL and apolipoprotein A (21).

\section{Tissue factor}

The tissue factor initiates the coagulation, increase platelet activity and contributes to the forming of thrombosis after plaque rupture (22).

\section{Endothelin-1}

Endothelin-1 contributes through its vasoconstrictor state to atherosclerosis pathogenesis. Furthermore, LDL oxidation causes an increase in the production and potentiation of endothelin 1 vasoconstrictor effects.

\section{Adhesion molecules}

Adhesion molecules are surface glycoproteins which mediates leucocyte adhesion to the endothelium. In atherosclerotic lesions there are high quantities of ICAM-1 and VCAM-1. These are reduced by the $\alpha$-tocopherol (vitamin E) through the reduction of $\beta 1$ and $\beta 2$ expression as well as by L-arginine which is the precursor of nitric oxide. Their increase is stimulated by androgens (23).

\section{Sanguine flux}

Disturbances in the sanguine flux can change the normal functioning of endothelial cells in such way that it also affects the atheroprotective function. These disturbances are mediated by the diminishing of nitric oxide from endothelial cells (20).

Other mechanisms proposed for atherosclerosis pathogenesis include mitochondrial DNA damage, genetics, and chronic infections such as Chlamydia pneumoniae, cytomegalovirus, coxsackie B virus and Helicobacter pylori.

\section{HISTOPATHOLOGY}

Atherosclerosis lesions involve the arterial intima. Initially focal thickening of the intima appear through accumulation of foam cells and extracellular matrix. Accumulation of lipids leads to the forming of fatty streaks. Furthermore, biglycan, which is a typical dermatan sulfate proteoglycan present in the atherosclerotic arteries intima, can bound lipoproteins such as VLDL and LDL. Later, the smooth muscle cells from deep layers of fatty streaks can become susceptible to apoptosis and associates with increased accumulation of macrophages which are predisposed to calcification thus, contributing to the progression of fatty streaks and atherosclerosis plaque. Fatty streaks with conjunctive tissue deposits, heightened number of smooth muscle cells and extracellular lipid deposits, progress towards the fibrous plaque. Complex plaques have a large lipidic core and inside this core calcification and hemorrhage can occur.

\section{CONCLUSIONS}

Plaque vulnerability appears when the stenosis obstructs $70-80 \%$ from the luminal diameter and it becomes manifested. Decrease in blood flow causes PAD specific symptoms with intermittent claudication.

Patients with diabetes mellitus are asymptomatic or have mild symptoms which hinder the discovery of atherosclerotic lesions while at the same time increasing the evolution potential towards more severe forms of PAD, up until amputation.

Control of the factors that contribute to the development and progression of atherosclerosis in diabetic patients will reduce the risk of PAD complication and improve the quality of life.

\section{Acknowledgement}

All authors contributed equally to the present work and thus are main authors.

Conflict of interest: none declared Financial support: none declared 


\section{REFERENCES}

1. Golden $\mathrm{SH}$, Anderson C, Bray GA, Burke LE, Boer IH De, Pignone M et al. Update on Prevention of Cardiovascular Disease in Adults With Type 2 Diabetes Mellitus in Light of Recent Evidence : A Scientific Statement From the American Heart Association and the American Diabetes Association. Diabetes Care. 2015 Sep;38(9):1777-803.

2. Jude $E$, Oyibo S, Chalmers N, Boulton a. Peripheral Arterial Disease in Diabetic and Nondiabetic Patients. Diabetes Care. 2001;24(8):1433-7.

3. Marso SP, Hiatt WR. Peripheral arterial disease in patients with diabetes. J Am Coll Cardiol. 2006;47(5):921-9.

4. Cantin B, Moorjani S, Dagenais GR, Lupien PJ. Lipoprotein(a) distribution in a French Canadian population and its relation to intermittent claudication (The Quebec Cardiovascular Study). Am J Cardiol. 1995;75(17):1224-8.

5. Bash LD, Selvin E, Steffes M, Coresh J, Astor BC. Poor glycemic control in diabetes and the risk of incident chronic kidney disease even in the absence of albuminuria and retinopathy: Atherosclerosis Risk in Communities (ARIC) study. Arch Intern Med. 2008;168(22):2440-7.

6. Newman JD, Schwartzbard AZ, Weintraub HS, Goldberg IJ, Berger JS. Primary Prevention of Cardiovascular Disease in Diabetes Mellitus. J Am Coll Cardiol. 2017;70(7):883-93

7. Criqui MH, Heiss G, Cohn R, Cowan LD, Suchindran CM, Bangdiwala S, Kritchevsky S, Jacobs DR Jr, O'Grady HK. Plasma triglyceride level and mortality from coronary heart disease. N Engl J Med. 1993;328(17):1220-5.

8. Park YM, Kashyap SR, Major JA, Silverstein RL. Insulin promotes macrophage foam cell formation: Potential implications in diabetes-related atherosclerosis. Lab Invest. 2012;92(8):1171-80.

9. Brownlee M. Biochemistry and molecular cell biology of diabetic complications. Nature. 2001;414(6865):813-20.

10. Bui MN, Sack MN, Moutsatsos G, Lu DY, Katz P, McCown R et al. Autoantibody titers to oxidized low-density lipoprotein in patients with coronary atherosclerosis. $\mathrm{Am}$ Heart J. 1996;131(4):663-7.

11. Inoue $T$, Uchida $T$, Kamishirado $H$, Takayanagi K, Hayashi T, Morooka S. Clinical significance of antibody against oxidized low density lipoprotein in patients with atherosclerotic coronary artery disease. J Am Coll Cardiol. 2001;37(3):7759.

12. Cho T, Romagnuolo R, Scipione C, Boffa MB, Koschinsky ML. Apolipoprotein (a) stimulates nuclear translocation of Bcatenin: A novel pathogenic mechanism for lipoprotein(a). Mol Biol Cell. 2013;24(3):210-21.

13. Li H, Förstermann U. Nitric oxide in the pathogenesis of vascular disease. J Pathol. 2000;190(3):244-54.

14. Herrmann J, Wilson SH, Ritmen EL, Holmes DR, Lilach O. Simvastatin Preserves the Structure of Coronary Advential Vasa Vasorum in Experimental Hypercholesterolemia Independent of Lipid Lowering. J Am Coll Cardiol. 2002;38:258.

15. Lindeman S, Gierer C, Darius H. Prostacyclin inhibits adhesion of polymorphonuclear leukocytes to human vascular endothelial cells due to adhesion molecule independent regulatory mechanisms. Basic Res Cardiol. 2003;98(1):8-15.

16. Ridker PM, Cushman M, Stampfer MJ, Tracy RP, Hennekens CH, Ridker PM, et al. Plasma concentration of $\mathrm{C}$-reactive protein and risk of developing peripheral vascular disease. Circ J Am Hear Assoc. 1998;97(5):425-8.

17. Escobar C, Blanes I, Ruiz A, Vinuesa D, Montero M, Rodríguez M et al. Prevalence and clinical profile and management of peripheral arterial disease in elderly patients with diabetes. Eur J Intern Med. 2011;22(3):275-81.

18. Fowkes FGR, Rudan D, Rudan I, Aboyans V, Denenberg JO, McDermott MM, et al. Comparison of global estimates of prevalence and risk factors for peripheral artery disease in 2000 and 2010: A systematic review and analysis. Lancet. 2013;382(9901):1329-40.

19. Geraldes P, King GL. Activation of Protein Kinase $C$ Isoforms and Its Impact on Diabetic Complications. Circ Res. 2010;106(8):1319-31.

20. Breen DM, Giacca A. Effects of Insulin on the Vasculature. Curr Vasc Pharmacol. 2011;9:321-32.

21. Greaves M, Malia RG, Goodfellow K, Mattock M, Stevens LK, Stephenson JM et al. Fibrinogen and von Willebrand factor in IDDM : Relationships to lipid vascular risk factors, blood pressure, glycaemic control and urinary albumin excretion rate : The EURODIAB IDDM complications study. Diabetologia 1997;698-705.

22. Inik AAI V. Platelet Dysfunction in Type 2 Diabetes. Diabetes Care. 2001;24:1476-85.

23. McCrohon JA, Jessup W, Handelsman DJ, Celermajer DS. Androgen exposure increases human monocyte adhesion to vascular endothelium and endothelial cell expression of vascular cell adhesion molecule-1. Circulation. 1999;99(17):231722. 